\title{
Queso de alpaca: una nueva alternativa
}

\author{
Alpaca Cheese: a new alternative \\ Heinz Larico M. ${ }^{1}$, Eliseo Fernández R. ${ }^{2}$, Ceferino Olarte D. ${ }^{3}$, Yésica Rodrigo V. ${ }^{1}$, \\ Pedro Machaca T. ${ }^{1}$, Regina Sumari M. ${ }^{1}$, Heber Chui B. ${ }^{1}$, Bernardo Roque H. ${ }^{1,4}$
}

\section{Resumen}

El trabajo tuvo como objetivo evaluar la posibilidad de elaborar queso a partir de leche de alpaca. Se determinaron las propiedades fisicoquímicas de la leche, así como el rendimiento, composición química y características sensoriales del queso. La leche se obtuvo por ordeño manual de alpacas madres en inicio de lactación con crías en pie, apartadas 12 horas antes del ordeño. En la leche se analizó la densidad, acidez Dornic, grasa Gerber, y se estimaron los sólidos totales y no grasos de la leche. La composición química del queso se determinó por métodos oficiales de la AOAC, y las características sensoriales del queso a través de la prueba hedónica en escala de 1 a 4 con 100 panelistas. La leche fresca presentó una densidad de $1.0469 \pm 0.0025$, acidez $16.92 \pm 1.74^{\circ} \mathrm{D}$, grasa $4.12 \pm 0.08 \%$, proteína $6.34 \pm 0.41 \%$, sólidos totales $16.84 \pm 0.70 \%$, sólidos no grasos $12.72 \pm 0.63 \%$ y un rendimiento quesero de $21.6 \pm 1.9 \%$. La composición química del queso fue humedad $46.5 \%$, ceniza $18.9 \%$, proteína $41.8 \%$, grasa $24.4 \%$ (en materia seca). El queso resultó un producto magro, de textura dura y agradable al consumo.

Palabras clave: leche; leche de alpaca; queso, queso de alpaca; rendimiento quesero

\section{AbSTRaCT}

The objective of this study was to evaluate the possibility of making cheese from alpaca milk. The physicochemical properties of the milk were determined, as well as the yield, chemical composition, and sensorial characteristics of the cheese. Milk was obtained by manual milking of alpacas at the beginning of lactation with crias at foot, set aside 12

\footnotetext{
${ }^{1}$ Laboratorio de Investigación de Camélidos, Facultad de Medicina Veterinaria y Zootecnia, Universidad Nacional del Altiplano, Puno, Perú

${ }^{2}$ Centro de Investigación Fundo Carolina, Universidad Nacional del Altiplano, Puno, Perú

${ }^{3}$ Instituto de Investigación y Promoción de Camélidos, Universidad Nacional del Altiplano, Puno, Perú

${ }^{4}$ E-mail: beroqueh@yahoo.es
}

Recibido: 20 de diciembre de 2017

Aceptado para publicación: 23 de mayo de 2018 
hours before milking. The density, Dornic acidity and Gerber fat were determined, and total and non-fat milk solids were estimated in the milk. The chemical composition of the cheese was determined by official methods of the AOAC, and the sensory characteristics of the cheese through the hedonic test on a scale of 1 to 4 with 100 panelists. Fresh milk had a density of $1.0469 \pm 0.0025$, acidity $16.92 \pm 1.74{ }^{\circ} \mathrm{D}$, fat $4.12 \pm$ $0.08 \%$, protein $6.34 \pm 0.41 \%$, total solids $16.84 \pm 0.70 \%$, solids non-fat $12.72 \pm 0.63 \%$ and a cheese yield of $21.6 \pm 1.9 \%$. The chemical composition of the cheese indicated $46.5 \%$ humidity, $18.9 \%$ ash, $41.8 \%$ protein, and $24.4 \%$ fat (dry matter). The cheese was a lean product with a hard texture and pleasant for consumption.

Key words: milk; alpaca milk; cheese; alpaca cheese; cheese yield

\section{INTRODUCCIÓN}

El Perú es uno de los países con mayor prevalencia de hambre en América Latina y El Caribe (FAO, 2011); así mismo, es uno de los más vulnerables a los efectos del calentamiento global y cambio climático (Rabatel et al., 2013), con repercusión en la producción de alimentos, por lo que está obligado a investigar alternativas de producción en este cambiante escenario.

La leche de camella se ha convertido en una alternativa importante para la seguridad alimentaria de los pueblos desérticos de África y Asia (Guliye et al., 2007; Mehari et al., 2007; Faye, 2013). Como alimento, es la leche más cercana a la leche materna humana (Zibaee et al., 2015), debido a su parecido en el perfil de ácidos grasos (Gul et al., 2015), mayor concentración de $\beta$-caseína y menor concentración de $\kappa-\mathrm{y} \alpha$-caseína que la leche de vaca (Salmen et al., 2012), falta de $\beta$ lacto-globulina y, por tanto, sin los problemas de alergia como ocurre con la leche de vaca (Merin et al., 2001; Shabo et al., 2005). A diferencia de la leche de vaca que contiene $\alpha$-lactosa, la leche de camella contiene $\beta$ lactosa de fácil digestión como la leche humana (Cardoso et al., 2010), posibilitando el desarrollo de bifidobacterias en el tracto intestinal (Roèková et al., 2013).
Su procesamiento en productos lácteos, tales como queso (Mehaia, 1993), yogurt y mantequilla (Jaeggi et al., 2005), ha posibilitado diversificar su consumo (Demissie et al, 2017); así como un importante apoyo en el control de los niveles de glucosa en sangre (Zibaee et al., 2015), gracias a su alta concentración de insulina (52 U/L) y a su facilidad para la absorción intestinal (Ahmed et al., 2013).

La llama y la alpaca son especies que tradicionalmente se han criado en los países andinos para la obtención de fibra y carne (Sumar, 1998); sin embargo, Fray Vicente de Valverde y Alonzo Gonzáles de Nájera describen en sus crónicas que los incas consumían leche y queso de llama (Dávila, 2007). Las leches de estas especies constituyen recursos subutilizados para las personas que viven en la zona andina (Fernández y Oliver, 1988). La leche de llama (Riek y Gerken, 2006) y de alpaca (Parraguez et al., 2003) son productos de alto valor nutricional que superan a la leche de otras especies de rumiantes en contenido de sólidos totales, proteína y grasa (Riek y Gerken, 2006); por consiguiente, pueden ofrecer excelente capacidad quesera, cono ocurre con la leche de camella (Yam y Khomeir, 2015).

Las alpacas pueden producir de 0.3 a 1 litro de leche por día, con densidad de 1.0351.050 , grasa $3-4 \%$ y $\mathrm{pH} 6.4-6.8$, aunque con 
alto coeficiente de variación (Moro, 1952), siendo necesario hacer selección genética para estos caracteres. En alpacas al pastoreo, la suplementación con heno de alfalfa mejoró la producción de leche durante las primeras siete semanas de lactación (Gonzáles et al., 2007).

La leche de alpaca tiene mayor contenido de sólidos totales (17.84\%), grasa $(6.40 \%)$ y lactosa $(5.70 \%)$ que la leche de vaca (López, 1974), menor contenido de ácidos grasos de cadena C14:0, mayor contenido de ácidos grasos insaturados y ácido linoleico conjugado (C18:2 cis-9, trans-11) (Martini et al., 2015). Las llamas, por su parte, producen $2.3 \mathrm{~kg}$ de leche diario entre la $3^{\text {ra }} \mathrm{y}$ $19^{\text {a }}$ semana posparto, con $15.6 \%$ de sólidos totales, grasa $4.7 \%$, proteína $4.2 \%$, lactosa $5.9 \%$ y N ureico $22.62 \mathrm{mg} / \mathrm{dl}$ (Riek y Gerken, 2006).

La leche de llama no contiene $\beta$-lacto globulina, proteína que constituye un factor de riesgo en el desarrollo de alergias en niños pequeños (Fernández y Oliver, 1988), siendo similar a la leche de camella (Merin et al., 2001; Shabo et al., 2005) y a la leche de mujer (El-Hatmi et al., 2015). Su grasa tiene predominio de ácidos grasos C16:0, C18:1, C14:0 y C18:0, ácidos grasos trans (3 $\mathrm{g} / 100 \mathrm{~g}$ ) del total de ácidos grasos, principalmente $\mathrm{C} 18: 1$ trans-11) y pequeña cantidad de ácido linoleico conjugado $(0.4 \mathrm{~g} / 100 \mathrm{~g}$ del total de ácidos grasos), el cual tiene efectos protectores contra algunos tumores cancerosos y capacidad de normalizar el metabolismo de la glucosa (Schoos et al., 2008).

El presente trabajo tuvo como objetivo evaluar la posibilidad de elaborar queso a partir de la leche de alpaca, para lo cual se determinaron las características fisicoquímicas y el rendimiento quesero de la leche, así como la composición química y las características sensoriales del queso elaborado a partir de la leche de alpaca.

\section{Materiales y Métodos}

La leche se obtuvo por ordeño manual de 10 alpacas madres en inicio de lactación con cría al pie, procedentes de un sistema extensivo en pastos naturales de la asociación Festuca dolichophylla - Muhlenbergia fastigiata. Las crías fueron separadas de sus madres en la tarde (18:30) y las madres fueron ordeñadas manualmente al día siguiente a las 06:30. La frecuencia de ordeño fue cada dos días, durante 40 días, en un brete, con un periodo previo de acostumbramiento al manejo.

Los insumos para la elaboración de queso fueron: leche entera, fresca y pasteurizada de alpaca, cuajo comercial (quimosina) de uso para leche de vaca, y sal común. Así mismo, se utilizaron los materiales de uso común en quesería. La densidad se determinó a través de un lactómetro, la acidez por titulación Dornic, la grasa por volumetría de Gerber (Eastwood et al., 2017) y la proteína a través de nitrógeno Kjeldahl x 6.38 (Lynch y Barbano, 1999).

El contenido de sólidos totales y sólidos no grasos de la leche se estimó a partir de la densidad de Quevenne, corregida a $15.5^{\circ} \mathrm{C}$, y el contenido porcentual de grasa Gerber en la leche, a través de la fórmula de Selvaggi et al. $(2017): \mathrm{ST} \%=0.25 \mathrm{D}+1.21 \mathrm{G}+0.14$; $\mathrm{SNG} \%=0.25 \mathrm{D}+0.21 \mathrm{G}+0.14$, donde $\mathrm{D}=$ densidad Quevenne corregida de la leche, $\mathrm{G}$ $=$ contenido porcentual de grasa Gerber en la leche.

El queso fue elaborado con adecuación al flujo de elaboración de queso de leche de vaca y camella (Shabo et al., 2005b), que consiste en la purificación de la leche por depuración física con un filtro, pasteurización a $60{ }^{\circ} \mathrm{C}$ por $30 \mathrm{~min}$, cortada con cuajo comercial a $38^{\circ} \mathrm{C}$ de temperatura (cuajo $1 \mathrm{~g} / 100 \mathrm{ml}$ de agua, cuajo diluido $2 \mathrm{ml} / 100 \mathrm{ml}$ de leche), 
primer corte de la cuajada a $1 \mathrm{~cm}$ de tamaño, segundo corte a tamaño de grano de arroz, calentado a $40{ }^{\circ} \mathrm{C}$, primer prensado por 30 min con $5 \mathrm{~kg}$, segundo prensado por $10 \mathrm{~h}$ con 5 $\mathrm{kg}$, y finalmente salmuera al 20\% durante $6 \mathrm{~h}$.

El rendimiento quesero de la leche se determinó mediante la siguiente fórmula: Rendimiento de queso $(\%)=($ Queso obtenido, $\mathrm{g}) /($ leche utilizada, $\mathrm{ml}) \times 100$, expresada en gramos de cuajada obtenida por mililitros de leche utilizada. La composición química del queso se determinó mediante los métodos oficiales de la AOAC (1999), en términos de materia seca, grasa total (extracto etéreo), proteína cruda y ceniza total.

Las características sensoriales del queso fueron determinadas mediante una prueba de aceptación a cargo de 100 panelistas. Se utilizó una escala hedónica de cuatro puntos (1: desagradable, 4: muy agradable), para evaluar olor, sabor, textura y nivel de aceptación en general, con adecuación a la evaluación sensorial utilizada para queso de camella y vaca (Mehaia, 1993; Siddig et al., 2016), en las instalaciones del Laboratorio de Nutrición Animal de la Facultad de Medicina Veterinaria y Zootecnia de la Universidad Nacional del Altiplano de Puno.

\section{Resultados y Discusión}

Las características fisicoquímicas de la leche de un grupo de alpacas en un día de ordeño se muestran en el Cuadro 1. Los valores medios obtenidos están de acuerdo con aquellos reportados para la leche de llama y alpaca (Parraguez et al., 2003; Riek y Gerken, 2006). El contenido de grasa, proteína, sólidos totales y sólidos no grasos de la leche de alpaca resultó más alto que los reportados para la leche de vaca, oveja y cabra (Dandare et al., 2014).

La producción de leche en llamas, estimada mediante técnica de dilución isotópica, varía desde $2.7 \mathrm{~kg} /$ día en la semana 3 hasta
$2.0 \mathrm{~kg}$ /día en la semana 18 posparto (Riek y Gerken, 2006), lo cual evidencia que los camélidos pueden producir tanta leche como cierto tipo de cabras, que con $15-20 \mathrm{~kg}$ de peso corporal producen $2.0 \mathrm{~kg}$ de leche por día (Shkolnik et al., 1980).

El contenido de sólidos totales y sólidos no grasos superan también los valores reportados para vacas y cabras (Fatima et al., 2013). A pesar de la discrepancia entre la determinación y la estimación en el presente estudio, el contenido estimado de sólidos totales y sólidos no grasos de la leche de alpaca supera ampliamente a aquellos en la leche de vaca o cabra (Kapadiya et al., 2016).

La densidad de la leche de alpaca (Cuadro 1) es también superior a la densidad de la leche de camella (Imran et al., 2008) y de otras especies, como consecuencia de su alto contenido en sólidos totales y sólidos no grasos. La acidez Dornic $\left(16.9 \pm 1.7^{\circ} \mathrm{D}\right)$ fue menor que la reportada para la leche de camella y de vaca (Mennane et al., 2007), similar a la de cabra y mucho menor que la de leche de oveja (Fatima et al., 2013).

Las diferencias en acidez pueden atribuirse a las características propias de la leche en cada especie, al periodo de lactación y al tipo de alimentación; sin embargo, el factor más importante es el ácido láctico que genera la fermentación de la lactosa por las bacterias que se agregan durante o después del ordeño (Widyastuti y Rohmatussolihat, 2014). Por lo general, la leche es un líquido virtualmente estéril cuando es secretada en el alveolo de la glándula mamaria; sin embargo, después ocurre contaminación microbiana desde diferentes fuentes (Mennane et al., 2007); por consiguiente, la baja acidez de la leche encontrada en este estudio evidencia higiene en el ordeño, manejo y conservación.

El contenido de grasa de la leche de alpaca está en el rango de valores reportados para leche de llama y alpaca (Parraguez et al., 2003; Khan et al., 2004; Hailu et al., 
Cuadro 1. Características fisicoquímicas de la leche de alpaca ( $\mathrm{n}=20$ quesos)

\begin{tabular}{lcccc}
\hline Variable & Promedio & $\begin{array}{c}\text { Desviación } \\
\text { estándar }\end{array}$ & Mínimo & Máximo \\
\hline Densidad & 1.04679 & 0.0025 & 1.0454 & 1.0483 \\
Sólidos totales, $\%^{1}$ & 16.84 & 0.70 & 16.43 & 17.25 \\
Sólidos no grasos, $\%^{2}$ & 12.72 & 0.63 & 12.35 & 13.09 \\
Acidez Dornic $\left({ }^{\circ} \mathrm{D}\right)$ & 16.92 & 1.74 & 15.9 & 17.9 \\
Grasa Gerber, \% & 4.12 & 0.08 & 4.07 & 4.17 \\
Proteína cruda, \% & 6.34 & 0.41 & 6.10 & 6.58 \\
\hline
\end{tabular}

${ }^{1}$ Estimado mediante fórmula: ST, $\%=0.25 \mathrm{D}+1.21 \mathrm{G}+0.14$

${ }^{2}$ Estimado mediante fórmula: SNG, \% $=0.25 \mathrm{D}+0.21 \mathrm{G}+0.14$ (Richmond, 1985)

Cuadro 2. Rendimiento y composición química del queso de alpaca ( $\mathrm{n}=20$ quesos)

\begin{tabular}{lcccc}
\hline Variable & Promedio & $\begin{array}{c}\text { Desviación } \\
\text { estándar }\end{array}$ & Mínimo & Máximo \\
\hline Leche utilizada, kg/día & 1.075 & 0.345 & 0.942 & 1.208 \\
Rendimiento de queso, \% & 21.6 & 1.9 & 20.9 & 22.3 \\
\hline Composición del queso & & & & \\
\hline Materia seca, \% & 53.5 & 1.5 & 52.4 & 54.6 \\
Ceniza, \% & 18.9 & 3.0 & 16.8 & 21.1 \\
Proteína cruda, \% & 41.8 & 3.7 & 39.0 & 44.5 \\
Grasa total, \% & 24.5 & 3.3 & 22.1 & 26.8 \\
\hline
\end{tabular}

2014) y de camella (Hailu et al., 2014); sin embargo, se observan diferencias con leche de otros rumiantes debido a su alta variabilidad. Así, entre 3.3 y $6.9 \%$ en vacas (Morvely, 1999; Auldist et al., 2004), 2.6-5.4\% en cabras (Soryal et al., 2005; Zullo et al., 2005), 2.4-10.4\% en ovejas (Bencini, 2002; Jaeggi et al., 2005; Ochoa et al., 2009). Las diferencias pueden atribuirse a que la grasa es el componente más variable de la leche, asociado a factores genéticos y ambientales (Selvaggi et al., 2017).
El contenido de proteína de la leche de alpaca (Cuadro 1) está en el rango reportado para la alpaca (Parraguez et al., 2003), pero superior al 2.1-5.6\% observado en leche de camella (Inayat et al., 2003; Abdoun et al., 2007; Hailu et al., 2014); así como al de otros rumiantes. Por ejemplo la cabra con 2.4-5.1\% (Soryal et al., 2005; Zullo et al., 2005), la vaca con 3.0-4.1\% (Auldist et al., 2004) y la oveja con 3.7-9.3\% (Bencini, 2002; Jaeggi et al., 2005). Las diferencias pueden atribuirse al factor genético, que define la capacidad de síntesis de la glándula mamaria en cada especie (Bionaz y Loor, 2011). 
Cuadro 3. Características sensoriales del queso de alpaca

\begin{tabular}{|c|c|c|}
\hline & Percepción & $\begin{array}{c}\text { Aceptación } \\
(\%)\end{array}$ \\
\hline Olor & $\begin{array}{l}\text { Agradable / } \\
\text { muy agradable }\end{array}$ & 74 \\
\hline Sabor & $\begin{array}{l}\text { Agradable / } \\
\text { muy agradable }\end{array}$ & 56 \\
\hline Textura & Dura & 65 \\
\hline
\end{tabular}

\section{Rendimiento Quesero}

La cantidad de leche disponible por día para la fabricación de 20 quesos fue de 1075 $\pm 345 \mathrm{ml}$. La leche formó una cuajada firme de 179.08 g, evidenciando buena capacidad quesera. El rendimiento estuvo en el rango de rendimiento quesero de la leche de otras especies lecheras $(21.6 \pm 1.9 \%$, Cuadro 2$)$, por ejemplo, $12.7 \%$ para leche de vaca (Morvely, 1999), 16\% para leche de cabra (Oliszewski et al., 2002), 21.4\% para leche de oveja (Morvely, 1999) y $26 \%$ para leche de camella (Cardoso et al., 2010).). Puesto que el queso concentra la proteína y la grasa de la leche, el rendimiento quesero se relaciona normalmente con el contenido de caseína y grasa, que son los mayores componentes de los sólidos totales de la leche (Imran et al., 2008).

\section{Composición Química del Queso}

El queso de alpaca es un producto de bajo contenido de humedad y alto contenido de materia seca (Cuadro 2). El bajo contenido de humedad puede atribuirse a las condiciones ambientales de la gran altitud que se caracteriza por una humedad relativamente baja. El queso de alpaca supera ampliamente al queso de camella en contenido de grasa, proteína y cenizas (Khan et al., 2004; Ahmed et al., 2013; Hailu et al., 2014); sin embargo, es bajo con relación al queso producido con leche de otras especies (Ahmed et al., 2013).
Dado que el contenido de materia seca es el referente para la clasificación de los quesos en duros, blandos o frescos, y considerando que los quesos duros contienen entre 52 y $54 \%$ de materia seca, el queso de alpaca puede ser considerado como un queso duro, con un contenido de materia seca que supera al del queso de camella (Khan et al., 2004; Hailu et al., 2014). Por otro lado, la caseína es el componente más importante del queso (Walther et al., 2008); por consiguiente, el producto elaborado en este estudio constituye una buena fuente de proteínas y aminoácidos esenciales para la nutrición.

\section{Características Sensoriales del Queso}

El resultado de la evaluación sensorial del queso de alpaca se muestra en el Cuadro 3. De los 100 panelistas, más de la mitad calificó favorablemente el queso, siendo la característica mejor apreciada el olor, seguida de la textura y el sabor.

Los sabores volátiles juegan un papel importante en la percepción del sabor del queso. El aroma típico del queso es el resultado de volátiles formados por la lipólisis, proteólisis y el metabolismo de la lactosa, lactato y citrato (McSweeney y Sousa, 2000). La textura del queso es uno de los determinantes de la opinión general y preferencia de los consumidores (Foegeding et al., 2013), y esta característica depende del contenido de grasa, de manera que los quesos pueden clasificarse en grasos o magros. Los quesos magros, por lo general, desarrollan una textura firme y sabor desagradable que puede dificultar el consumo; sin embargo, el queso de alpaca, sometido a prueba de degustación, tan pronto como fue madurado, tuvo una alta aceptación (65\%) por los panelistas (Cuadro 3 ), superior a la aceptación reportada para el queso de camella (Ahmed et al., 2013).

La leche de camella y sus derivados se han convertido en parte de la seguridad alimentaria de los pueblos del desierto (Faye, 2014). El queso de burra es el queso más caro (Iannella, 2015), de allí que la leche o 
queso de llama o alpaca podrían constituirse en nuevas fuentes de alimentos no convencionales para la alimentación de la futura población (Floros et al., 2010).

\section{Conclusiones}

- La leche de alpaca tiene una densidad de 1.0468 , acidez $16.9^{\circ} \mathrm{D}, 6.3 \%$ de proteína y $4.1 \%$ de grasa.

- $\quad$ El rendimiento de queso fue de $21.6 \%$.

- El queso elaborado a partir de leche de alpaca contiene $46.5 \%$ de humedad, $24.4 \%$ de grasa, $41.8 \%$ de proteína y $18.9 \%$ de cenizas totales en materia seca, siendo un producto magro de textura dura y buena aceptación.

\section{Agradecimientos}

Los autores expresan su agradecimiento a la Universidad Nacional del Altiplano de Puno por el soporte económico. A los laboratorios de Bioquímica y Nutrición Animal, al Centro de Investigación y Producción Ciudad Universitaria, y al Fundo Carolina por el apoyo logístico.

\section{Literatura Citada}

1. Abdoun KA, Amin AS, Abdelatif AM. 2007. Milk composition of dromedary camels (Camelus dromedarius): nutritional effects and correlation to corresponding blood parameters. Pak J Biol Sci 10: 2724-2727. doi: 10.3923/ pjbs.2007.2724.2727

2. Ahmed A, Babiker IA, Mohamed TE. 2013. Preparation of fresh soft cheese from dromedary camel milk using acid and heat method. Res Opinion Anim Vet Sci 3: 289-292.

3. AOAC. 1999. Official methods of analysis of AOAC International. Cunniff P (ed). Washington, USA: Association of Official Analytical Chemists.
4. Auldist MJ, Johnston KA, White NJ, Fitzsimons WP, Boland MJ. 2004. A comparison of the composition, coagula-tion characteristics and cheesemaking capacity of milk from Friesian and Jersey dairy cows. J Dairy Res 71: 51-57.

5. Bencini RR. 2002. Factors affecting the clotting properties of sheep milk. J Sci Food Agr 82: 705-719. doi: 10.1002/ jsfa.1101

6. Bionaz M, Loor JJ. 2011. Gene networks driving bovine mammary protein synthesis during the lactation cycle. Bioinform Biol Insights 5: 83-98. doi: 10.4137/BBI.S7003

7. Cardoso RR, Santos RM, Cardoso CR, Carvalho MO. 2010. Consumption of camel's milk by patients intolerant to lactose. A preliminary study. Rev Alerg Mex 57: 26-32.

8. Dandare SU, Ezeonwumelu IJ, Abubakar MG. 2014. Comparative analysis of nutrient composition of milk from different breeds of cows. Europ J Appl Engin Sci Res 3(2): 33-36.

9. Dávila MJ. 2007. Consumo de la leche de llama (Lama glama) en los Andes peruanos. Cienc Des 8(1): 5-18. doi: 10.21503/CienciayDesarrollo.2007.v8.01

10. Demissie B, Komicha HH, Kedir A. 2017. Production and marketing of camel milk in Eastern Ethiopia. Afr J Mark Manage 9: 98-106.

11. Eastwood C, Klerkx L, Nettle R. 2017. Dynamics and distribution of public and private research and extension roles for technological innovation and diffusion: case studies of the implementation and adaptation of precision farming technologies. J Rural Stud 49: 1-12. doi: 10.1016/j.jrurstud.2016.11.008

12. El-Hatmi H, Jrad Z, Salhi I, Aguibi A, Nadri A, Khorchani T. 2015. The composition and whey protein fractions of the human, camel, donkey, goat and cow milk. Mljekarstvo 65: 159-167. doi: 10.15567/mljekarstvo.2015.0302

13. [FAO] Food and Agriculture Organization of the United Nations. 2011. World livestock 2011. Livestock in food security. Rome, Italy: FAO. 115 p. 
14. Fatima A, Kheira B, Bettache G, Habib A, Mebrouk K. 2013. Evaluation of microbiological and sanitary quality of ewe's raw milk in Western of Algeria and detection of antibiotic residue by Delvotest. Adv Environ Biol 7: 10271033.

15. Faye B. 2013. Camel farming sustainability: the challenges of the camel farming system in the XXI ${ }^{\text {th }}$ century. J Sustain Devel 6(12): 74-82. doi: 10.5539/ jsd.v6n12p74

16. Faye B. 2014. The camel today: assets and potentials. Anthropozoologica 49: 167-176. doi: 10.5252/az2014n2a01

17. Fernández FM, Oliver G. 1988. Proteins present in llama milk. Quantitative aspects and general characteristics. Milchwissenschaft 43: 299-302.

18. Floros J, Newsome W, Fisher GV, Barbosa-Canovas H, Chen CP, Dunne $J B$, German RL, et al. 2010. Feeding the world today and tomorrow: the importance of food science and technology. Compr Rev Food Sci F 9: 572-599. doi: 10.1111/j.1541-4337.2010.00127.x

19. Foegeding EA, Brown J, Drake M, Daubert CR. 2013. Sensory and mechanical aspects of cheese texture. Int Dairy J 13: 585-591. doi: 10.1016/ S0958-6946(03)00094-3

20. Gonzáles $R$, Leyva V, García W, Gavidia C, Ticona D. 2007. Efecto de la alimentación sobre la producción láctea en llamas seleccionadas para producción de carne. Rev Inv Vet Perú 18: 30 39. doi: 10.15381/rivep.v18i1.1272

21. Gul W, Farooq N, Anees D, Khan U, Rehan F. 2015. Camel milk: a boon to mankind. Int J Res Stud Biosci 3: 23-29.

22. Guliye AY, Noor IM, Bebe BO, Koskey IS. 2007. Role of camels (Camelus dromedarius) in the traditional lifestyle of Somali pastoralists in northern Kenya. Outlook Agr 36: 29-34.

23. Hailu Y, Seifu E, Yilma Z. 2014. Physicochemical properties and consumer acceptability of soft unripened cheese made from camel milk using crude extract of ginger (Zingiber officinale) as coagulant. Afr J Food Sci 8: 87-91.

24. Iannella G. 2015. Donkey cheese made through pure camel chymosin. Afr J Food Sci 9: 421-425.

25. Imran M, Khan H, Hassan SS, Khan R. 2008. Physicochemical characteristics of various milk samples available in Pakistan. J Zhejiang Univ Sci B 9: 546551. doi: 10.1631/jzus.B0820052

26. Inayat $S$, Arain MA, Khaskheli $M$, Malik AH. 2003. Study of the effect of processing on the chemical quality of soft unripened cheese made from camel milk. Pakistan J Nutr 2: 102-105. doi: 10.3923/ pjn.2003.102.105

27. Jaeggi JJ, Wendorff WL, Romero J, Berger YM, Johnson ME. 2005. Impact of seasonal changes in ovine milk on composition and yield of a hardpressed cheese. J Dairy Sci 88: 13581363. doi: 10.3168/jds.S0022-0302(05)72802-2

28. Kapadiya DB, Prajapati DB, Jain AK, Mehta BM, Darji VB, Aparnathi KD. 2016. Comparison of Surti goat milk with cow and buffalo milk for gross composition, nitrogen distribution, and selected minerals content. Vet World 9: 710-716. doi: 10.14202/vetworld.2016.710-716

29. Khan H, Hussain I, Athar IH, Aslam M. 2004. Evaluation of cheese prepared by processing camel milk. Pakistan J Zool 36: 323-326.

30. López J. 1974. Calidad de la leche de alpacas. En: II Reunión Nacional de Investigadores en Ganadería. La Paz, Bolivia.

31. Lynch JM, Barbano DM. 1999. Kjeldahl nitrogen analysis as a reference method for protein determination in dairy products. J AOAC Int 82: 1389-1398.

32. Martini MI, Altomonte AM, da Silva Santana G, del Plavignano G, Salaric F. 2015. Gross, mineral and fatty acid composition of alpaca (Vicugna pacos) milk at 30 and 60 days of lactation. Small 
Ruminant Res 132: 50-54. doi: 10.1016/ j.smallrumres.2015.10.001

33. McSweeney PLH, Sousa MJ. 2000. Biochemical pathways for the production of flavor compounds in cheese during ripening: a review. Lait 80: 293-324. doi: 10.1051/lait:2000127

34. Mehaia MA. 1993. Fresh soft white cheese (Domiati-type) from camel milk: composition, yield, and sensory evaluation. J Dairy Sci 76: 2845-2855. doi: 10.3168/jds.S0022-0302(93)77623-7

35. Mehari Y, Mekuriaw Z, Gebru G. 2007. Camel and camel product marketing in Babilie and Kebribeyah woredas of the Jijiga Zone, Somali region, Ethiopia. Livestock Res Rural Develop 19(49). [Internet]. Available in: http://www.1rrd.org/1rrd19/4/ meha19049.htm

36. Mennane Z, Ouhssine M, Khedid K, Elyachioui M. 2007. Hygienic quality of raw cow's milk feeding from domestic waste in two regions of Morocco. Int $\mathrm{J}$ Agri Biol 9(1): 46-48.

37. Merin U, Bernstein S, Bloch-Damti N, Yagil $R$, van Creveld $C$, Lindner $P$, Gollop N. 2001. A comparative study of milk proteins in camel (Camelus dromedarius) and bovine colostrum. Livest Prod Sci 67: 297-301. doi: 10.1016/ S0301-6226(00)00198-6

38. Moro M. 1952. Contribución al estudio de la leche de las alpacas. Rev Fac Med Vet UNMSM 7(11): 117-141.

39. Morvely CM. 1999. Características del queso semiduro elaborado con leche de oveja y/o vaca. Tesis de Médico Veterinario Zootecnista. Puno, Perú: Univ. Nacional del Altiplano. $90 \mathrm{p}$.

40. Ochoa A, Vega L, Ochoa M, Bisset P, Torres $G$ 2009. Características físicoquímicas de la leche de ovejas Rambouillet bajo manejo intensivo. Rev Cient (Maracaibo) 19: 196-200.

41. Oliszewski R, Rabasa AE, Fernández JL, Poli MA, Kairúz MS. 2002. Composición química y rendimiento quesero de la leche de cabra criolla serrana del noroeste argentino. Zootecnia Trop 20: 179-189.

42. Parraguez VH, Thénot M, Latorre E, Ferrando G, Raggi LA. 2003. Milk composition in alpaca (Lama pacos): comparative study in two regions of Chile. Arch Zootec 52: 431-439.

43. Rabatel A, Francou B, Soruco A. 2013. Current state of glaciers in the tropical Andes: a multi-century perspective on glacier evolution and climate change. Cryosphera 7: 81-102.

44. Richmond HD. 1985. The relation between specific gravity, fat, and solidsnot-fat in milk. Analyst 20: 57-58.

45. Riek A, Gerken M. 2006. Changes in llama (Lama glama) milk composition during lactation. J Dairy Sci 89: 34843493. doi: 10.3168/jds.S0022-0302(06)72387-6

46. Roèková Š, Rada V, Havlík J, Švejstil R, Vlková E, Bunešová V, et al. 2013. Growth of bifidobacteria in mammalian milk. Czech J Anim Sci 58: 99-105.

47. Salmen SH, Abu-Tarboush HM, AlSaleh AA, Metwalli AA. 2012. Amino acids content and electrophoretic profile of camel milk casein from different camel breeds in Saudi Arabia. Saudi J Biol Sci 19: 177-183. doi: 10.1016/j.sjbs.2011.12.002

48. Schoos V, Medina M, Saad S, van Nieuwenhove P. 2008. Chemical and microbiological characteristics of llamas' (Lama glama) milk from Argentina. Milchwissenschaft 63: 398-401.

49. Selvaggi M, D'Alessandro AG, Dario C. 2017. Environmental and genetic factors affecting milk yield and quality in three Italian sheep breeds. J Dairy Res 84: 27-31. doi: 10.1017/S0022029916000765

50. Shabo Y, Barzel R, Margoulis M, Yagil R. 2005a. Camel milk for food allergies in children. Isr Med Assoc J 7: 796-798.

51. Shkolnik A, Maltz E, Gordin S. 1980. Desert conditions and goat milk production. J Dairy Sci 63: 1749-1754. doi: 10.3168/jds.S0022-0302(80)83134-1 
52. Siddig SM, Sulieman AME, Salih ZA, Abdelmuhsin AA. 2016. Quality characteristics of white cheese (Jibnabeida) produced using camel milk and mixture of camel milk and cow milk. Int J Food Sci Nutr 6: 49-54. doi: 10.5923/ j.food.20160603.01

53. Soryal K, Beyene FA, Zeng S, Bah B, Tesfai K. 2005. Effect of goat breed and milk composition on yield, sensory quality, fatty acid concentration of soft cheese during lactation. Small Ruminant Res 58: 275-281. doi: 10.1016/j.smallrumres.2004.11.003

54. Sumar J. 1998. Present and potential role of South American camelids in the High Andes. Outlook Agric 17: 23-29.

55. Walther B, Schmid A, Sieber R, Wehrmuller K. 2008. Cheese in nutrition and health. Dairy Sci Technol 88:389-405.
56. Widyastuti Y, Rohmatussolihat, Febrisian-tosa A. 2014. The role of lactic acid bacteria in milk fermentation. Food Nutr Sci 5: 435-442.

57. Yam BAZ, Khomeir M. 2015. Introduction to camel origin, history, raising, characteristics, and wool, hair and skin: a review. Res J Agric Environ Manage 4: 496-508.

58. Zibaee S, Hosseini SM, Yousefi M, Taghipour A, Kiani MA, Noras MR. 2015. Nutritional and therapeutic characteristics of camel milk in children: a systematic review. Electron Physician 7: 1523-1528. doi: 10.19082/1523

59. Zullo A, Barone CMA, Chianese L, Colatruglio L, Occidente P, Matassino D. 2005. Protein polymorphisms and coagulation properties of Cilentana goat milk. Small Ruminant Res 58: 223-230. doi: 10.1016/j.smallrumres.2004.10.003 